\title{
Concerning collectionwise Hausdorff spaces
}

\section{James R. Boone}

In this paper the notion of a space which has property (w) pointwise is studied. The primary application of this concept is a reformulation of Tall's existence theorem for normal nonmetrizable metacompact Moore spaces in terms of families which are finite on convergent sequences. Since the first countability in Tall's' theorem yields an abundance of convergent sequences, this reformulation places the existence theorem in a natural setting.

\section{Preliminaries}

A topological space is collectionwise Hausdorff if for each discrete collection of points $\left\{p_{\alpha}: \alpha \in A\right\}$ there is a disjoint collection of open sets $\left\{U_{\alpha}: \alpha \in A\right\}$ such that $p_{\alpha} \in U_{\alpha}$, for each $\alpha \in A$. Tall [7] has reduced the question of the existence of a normal Moore space which is not metrizable to the following theorem.

THEOREM 1.1 [Tall]. If there is a normal first countable space which is not collectionwise Hausdorff, then there exists a normal nonmetrizable metacompact Moore space.

The set theoretic consequences of the answer to the Moore metrization problem are well documented in the various works of Tall. Tall's Theorem places this question in a highly simplified setting and reduces the consideration of the metrizability of normal metacompact Moore spaces, from discrete collections of closed sets to discrete sets of points.

Received 24 May 1974. 
The primary purpose of this paper is to present a reformulation of Tail's Theorem (Corollary 3.2) in terms of families which are finite on convergent sequences. For this purpose the notion of a space with property (w) pointwise is introduced.

\section{2, Definitions}

A space will be called strongly collectionvise Hausdorff if for each discrete set of points $\left\{p_{\alpha}: \alpha \in A\right\}$ there is a discrete collection of open sets $\left\{U_{\alpha}: \alpha \in A\right\}$ such that $p_{\alpha} \in U_{\alpha}$, for each $\alpha \in A$. Every normal collectionwise Hausdorff space is strongly collectionwise Hausdorff. A collection of subsets $G$ of a space $X$ is said to be cs-finite at $p$, $p \in X$, if for each sequence $\left\{p_{i}\right\}$ in $X$, such that $p_{i} \rightarrow p$, $G \cap\left\{p_{i}: i \in N\right\} \neq \varnothing$ for at most a finite number of $G \in G$. The collection $G$ is as-finite if it is cs-finite at every point in $X$. A space $X$ is said to have property ( $\omega$ ) [2] (pointwise collectionavise normal [3]) if for each discrete collection of closed sets $F=\left\{F_{\alpha}: \alpha \in A\right\}$, there exists a cs-finite (point-finite) collection of open sets $G=\left\{G_{\alpha}: \alpha \in A\right\}$ such that $F_{\alpha} \subset G_{\alpha}$, for each $\alpha \in A$ and $F_{\alpha} \cap G_{\beta}=\varnothing$, if $\alpha \neq \beta$. Every collectionwise normal space has property (w), and if a space has property (w) then it is pointwise collectionwise normal. Every metacompact space is pointwise collectionwise normal. A Moore space is metrizable if and only if it has property ( $\omega)$ [2]. Two of the results reported in [3] are:

a space is metacompact if and only if it is $\theta$-refinable [8] and pointwise collectionwise normal, and

a developable pointwise collectionwise normal space is hereditarily metacompact.

DEFINITION. A space is said to have property $(\omega)$ pointwise if for each discrete set of points $\left\{p_{\alpha}: \alpha \in A\right\}$ there exists a cs-finite collection of open sets $G=\left\{G_{\alpha}: \alpha \in A\right\}$ such that $p_{\alpha} \in G_{\alpha}$, for each $\alpha \in A$ and $p_{\alpha} \vDash G_{\beta}$, if $\alpha \neq B$.

Every space with property $(\omega)$ has property $(\omega)$ pointwise and 
every strongly collectionwise Hausdorff space has property $(\omega)$ pointwise.

\section{Theorems}

THEOREM 3.1. Every regular sequential space which has property (w) pointwise is strongly collectionaise Hausdorff.

Proof. Let $\left\{p_{\alpha}: \alpha \in A\right\}$ be any discrete set of points in a regular space $X$. Let $\left\{U_{\alpha}: \alpha \in A\right\}$ be a cs-finite collection of open sets such that $p_{\alpha} \in U_{\alpha}$ for each $\alpha \in A$ and $p_{\beta} \neq U_{\alpha}$, if $\beta \neq \alpha$. For each $\alpha \in A$, let $V_{\alpha}$ be an open set such that $p_{\alpha} \in V_{\alpha} \subset \operatorname{cl}\left(V_{\alpha}\right) \subset U_{\alpha}$. Then $\left\{c 1\left(V_{\alpha}\right): \alpha \in A\right\}$ is a cs-finite collection of closed sets in a sequential space. Accordingly, $\left\{\operatorname{cl}\left(V_{\alpha}\right): \alpha \in A\right\}$ is locally finite by $[1$, Lemma 2.1]. If $O_{\alpha}$ is an open subset of $X$ such that

$$
p_{\alpha} \in O_{\alpha} \subset \operatorname{cl}\left(O_{\alpha}\right) \subset V_{\alpha}-U\left\{\operatorname{cl}\left(V_{\beta}\right): \beta \neq \alpha\right\}
$$

for each $\alpha \in A$, then $\left(_{\alpha}: \alpha \in A\right\}$ is a discrete collection of open sets such that $p_{\alpha} \in O_{\alpha}$ for each $\alpha \in A$. This completes the proof.

COROLLARY 3.2. If there exists a normal first countable space which fails to have property ( $\omega$ ) pointwise, then there is a normal nonmetrizable metacompact Moore space.

Additional corollaries to Theorem 3.1 are apparent, for those theorems involving normal collectionwise Hausdorff spaces, in the papers of Fitzpatrick [5], Christian [4], and Tall [6].

THEOREM 3.3. Let $f: X \rightarrow Y$ be an open and closed continuous surjection defined on a pointwise collectionwise normal space $X$. If $X$ is sequential and has property $(\omega)$ pointwise, then $Y$ has property $(\omega)$ pointurise.

Proof. Since pointwise collectionwise normality is invariant under closed mappings [3], $Y$ is pointwise collectionwise normal. Let $\left\{p_{\alpha}: \alpha \in A\right\}$ be any discrete set in $Y$. There exists a point-finite collection of open sets, $V=\left\{V_{\alpha}: \alpha \in A\right\}$, such that $p_{\alpha} \in V_{\alpha}$, for each 
$\alpha \in A$ and $p_{\beta} \neq V_{\alpha}$, if $\beta \neq \alpha$. If $q_{\alpha} \in f^{-1}\left(p_{\alpha}\right)$ for each $\alpha \in A$, $\left\{q_{\alpha}: \alpha \in A\right\}$ is a discrete subset of $X$. There exists a cs-finite collection of open sets, $\left\{G_{\alpha}: \alpha \in A\right\}$, such that $q_{\alpha} \in G_{\alpha}$ for each $\alpha \in A$ and $q_{\alpha} \neq G_{\beta}$, if $\alpha \neq B$. For each $\alpha \in A$, let $U_{\alpha}=\left(f\left(G_{\alpha}\right)-\left\{p_{\gamma}: \gamma \neq \alpha, \gamma \in A\right\}\right) \cap V_{\alpha}$. Then $p_{\alpha} \in U_{\alpha}$, for each $\alpha \in A$ and $p_{\alpha} \neq U_{\beta}$, if $\alpha \neq B$. Let $u=\left\{U_{\alpha}: \alpha \in A\right\}$. Since $V$ is pointfinite, $U$ is point-finite. Assume $U$ is not cs-finite. There exists some convergent sequence $\left\{r_{i}\right\}$ in $Y$, say $r_{i} \rightarrow r$, such that $A^{\prime}=\left\{\alpha \in A: U_{\alpha} \cap\left\{r_{i}: i \in N\right\} \neq \emptyset\right\}$ is infinite. The point-finiteness of $U$ allows us to suppose $r_{i} \neq r$, for each $i \in N$. Let $i_{1}$ be the first index such that $r_{i_{1}} \in U_{\alpha_{1}}$, for some $\alpha_{1} \in A^{\prime}$. Since $u$ is pointfinite, there exists a second index $i_{2}$ such that $i_{1}<i_{2}$, and $r_{i_{2}} \in U_{\alpha_{2}}$ for some $\alpha_{2} \in A^{\prime}-\left\{\alpha_{1}\right\}$. Suppose this process has been continued $n-1$ times. That is, for each $j \in N$, such that $1<j \leq n-1, i_{j}$ is an index such that $i_{1}<i_{2}<\ldots<i_{j}$ and $r_{i j} \in U_{\alpha_{j}}$ for some $\alpha_{j} \in A^{\prime}-\left\{\alpha_{1}, \alpha_{2}, \ldots, \alpha_{j-1}\right\}$. Since $u$ is pointfinite, there is an index $i_{n}$, such that $i_{1}<i_{2}<\ldots<i_{n-1}<i_{n}$ and $r_{i_{n}} \in U_{\alpha_{n}}$ for some $\alpha_{n} \in A^{\prime}-\left\{\alpha_{1}, \alpha_{2}, \ldots, \alpha_{n-1}\right\}$. Then $\left\{r_{i_{n}}\right\}$ is a subsequence of $\left\{r_{i}\right\}, r_{i_{n}} \in U_{\alpha_{n}}$ for each $n \in N$, and $U_{\alpha_{n}} \neq U_{\alpha_{k}}$, if $k \neq n$. Since $r_{i_{n}} \in U_{\alpha_{n}} \subset f\left(G_{\alpha_{n}}\right)$, for each $n \in N, f^{-1}\left(r_{i_{n}}\right) \cap G_{\alpha_{n}} \neq \emptyset$. Let $t_{i_{n}} \in f^{-1}\left(r_{i_{n}}\right) \cap G_{\alpha_{n}}$, for each $n \in N$. Then $\left\{t_{i_{n}}: n \in N\right\}$ is not closed, because $\left\{r_{i_{n}}: n \in N\right\}$ is not closed. Since $X$ is sequential, $\left\{t_{i_{n}}: n \in N\right\}$ is not sequentially closed. Hence, there exists a sequence in $\left\{t_{i_{n}}: n \in N\right\}$ which converges to some point in $f^{-1}(r)$. This 
sequence must intersect infinitely many of the sets $G_{\alpha_{n}}$, contradicting the cs-finiteness of $\left\{G_{\alpha}: \alpha \in A\right\}$. Accordingly, $U$ is cs-finite. This completes the proof. Note that if $Y$ is regular, then $Y$ is strongly collectionwise Hausdorff, because $Y$ is sequential.

THEOREM 3.4. The closed and open continuous image of a normal collectionwise Hausdorff space is a normal collectionwise Hausdorff space.

Proof. Let $f: X \rightarrow Y$ be a closed and open continuous surjection defined on a normal collectionwise Hausdorff space $X$. Let $\left\{p_{\alpha}: \alpha \in A\right\}$ be a discrete set in $Y$. If $q_{\alpha} \in f^{-1}\left(p_{\alpha}\right)$ for each $\alpha \in A$, then $\left\{q_{\alpha}: \alpha \in A\right\}$ is a discrete set in $X$. Let $u=\left\{U_{\alpha}: \alpha \in A\right\}$ be a discrete collection of open subsets of $X$ such that $p_{\alpha} \in U_{\alpha}$ for each $\alpha \in A$. Since $f$ is closed, $Y$ is normal and for each $\alpha \in A$ we let $G_{\alpha}$ be an open subset of $Y$ such that

$$
p_{\alpha} \in G_{\alpha} \subset \operatorname{cl}\left(G_{\alpha}\right) \subset f\left(U_{\alpha}\right)-\left\{p_{\beta}: \beta \neq \alpha, \beta \in A\right\} \text {. }
$$

Since $U$ was discrete, $\left\{f\left(U_{\alpha}\right): \alpha \in A\right\}$ is hereditarily closure preserving. Accordingly, if $V_{\alpha}=G_{\alpha}-U\left\{c l\left(G_{\beta}\right): \beta \neq \alpha, \beta \in A\right\}$ for each $\alpha \in A$, then $\left\{V_{\alpha}: \alpha \in A\right\}$ is a disjoint collection of open subsets of $Y$ such that $p_{\alpha} \in V_{\alpha}$ for each $\alpha \in A$. This completes the proof.

THEOREM 3.5. In a first countable space, the set of points, $S$, at which a point-finite collection of sets $G=\left\{G_{\alpha}: \alpha \in A\right\}$ fails to be cs-finite is precisely the set of points at which $G$ fails to be locally finite. Thus $S$ is closed.

Proof. Let $p$ be any point in $S$, and let $\left\{p_{i}\right\}$ be such that $p_{i} \rightarrow p$ and $G_{\alpha} \cap\left\{p_{i}: i \in N\right\} \neq \emptyset$ for infinitely many $\alpha \in A$. Since $G$ is point-finite, there exists a subsequence $\left\{p_{i_{n}}\right\}$ of $\left\{p_{i}\right\}$ such that $p_{i_{n}} \in G_{\alpha_{n}}$, for each $n \in N$ and $G_{\alpha_{n}} \neq G_{\alpha_{k}}$, if $n \neq k$. Since $p_{i_{n}} \rightarrow p$, every neighborhood of $p$ intersects infinitely many different $G_{\alpha_{n}} \in G$. 
Hence $G$ is not locally finite at $p$. To prove the converse, let $p$ be any point at which $G$ is not locally finite, and let $\left\{U_{i}: i \in N\right\}$ be any countable neighborhood base at $p$, in which $U_{i+1} \subset U_{i}$, for each $i \in N$. For $i=1$, let $\alpha_{1} \in A$, such that $U_{1} \cap G_{\alpha_{1}} \neq \varnothing$. Let $p_{1} \in \dot{U}_{1} \cap G_{\alpha_{1}}$. Suppose that for each $i, 1 \leq i \leq n-1, p_{i} \in U_{i} \cap G_{\alpha_{i}}$ and $G_{\alpha_{i}} \neq G_{\alpha_{k}}$, for each $k, 1 \leq k<i$. Since $G$ is not locally finite at $p$, $U_{n} \cap G_{\alpha_{n}} \neq \emptyset$, for some $G_{\alpha_{n}} \neq G_{\alpha_{k}}$, for each $k, 1 \leq k \leq n-1$. Let $p_{n} \in U_{n} \cap G_{\alpha_{n}}$. The sequence $\left\{p_{n}\right\}$ converges to $p$ and $\left\{p_{n}: n \in N\right\} \cap G_{\alpha} \neq \emptyset$, for infinitely many $G_{\alpha} \in G$. Thus $p \in S$. It is clear that the set of points at which a collection fails to be locally finite is closed. This completes the proof.

\section{References}

[1] James R. Boone, "Some characterizations of paracompactness in k-spaces", Frond. Math. 72 (1971), 145-153.

[2] James R. Boone, "A metrization theorem for developable spaces", Fund. Math. 73 (1971), 79-83.

[3] James R. Boone, "A characterization of metacompactness in the class of 0-refinable spaces", General Topology and Appl. 3 (1973), 253-264.

[4] U.J. Christian, "Concerning certain minimal cover refinable spaces", Fund. Math. 76 (1972), 213-222.

[5] Ben Fitzpatrick, Jr., "On dense subspaces of Moore spaces II", Fund. Math. 61 (1967), 91-92.

[6] Franklin D. Tall, "Set-theoretic consistency results and topological theorems concerning the normal Moore space conjecture and related problems", $\mathrm{PhD}$ thesis, University of Wisconsin, Madison, 1969.

[7] Franklin D. Tall, "On the existence of normal metacompact Moore spaces which are not metrizable", Cconad. J. Math. 26 (1974), 1-6. 
[8] J.M. Worrell, Jr. and H.H. Wicke, "Characterizations of developable topological spaces", Canad. J. Math. 17 (1965), 820-830.

Department of Mathematics,

Texas A\&M University,

College Station,

Texas,

USA. 